\title{
Multi-Scale Adaptive Gain Control of IR Images
}

\author{
K. Schutte \\ TNO Physics and Electronics Laboratory \\ P.O. Box 96864, 2509 JG The Hague, The Netherlands \\ Email: Schutte@fel.tno.nl
}

\begin{abstract}
IR imagery tends to have a higher dynamic range then typical display devices such as a CRT. Global methods such as stretching and histogram equalization improve the visibility of many images, but some information in the images stays hidden for a human operator. This paper reports about the possibility to represent more information by using local adaptive techniques. Based upon methods found in literature, a new multi-scale method is presented.
\end{abstract}

Keywords: Gain Control, Image Enhancement, Local Image Statistics

\section{LITERATURE}

In literature several methods of local adaptive enhancement of images are reported. All are based on the principal of acquiring the local statistics of the region under study, and acting upon those statistics and the value of the pixel itself.

Two different methods are used in literature for describing the local statistics of an image region. The first method $^{1,2}$ to describe these statistics is to characterize it by its mean and variance. An adaptive scheme based on the local variance and mean is used to add the difference between mean and the original pixel value to the result. The second method ${ }^{3,4}$ to describe the local statistics is to calculate the histogram of the neighborhood. Using that histogram, histogram equalization is applied to the center pixel of the neighborhood. This leads to solutions where the output pixel value only depends on the local statistics, and no longer on global values; and thus it becomes in some situations harder to distinguish clutter from real objects. Also, histogram based methods tend to be computationally more intensive.

Besides the methods described above, Toet ${ }^{5}$ described a multi-scale approach to contrast enhancement based on a non-linear pyramid recombination.

In both Ref. 1 and Ref. 2 the improvement of the image is obtained by using a function like:

$$
o=i+f .(i-m)
$$

with $i$ the input pixel value, $o$ the output pixel value, $m$ the local mean and $f$ a function depending on the local statistics. In Ref. 2 care is taken that already large contrasts are not enhanced too much, where in Ref. 1 this function also takes care not to amplify background noise.

The difference between standard local histogram equalization procedures (see for example Gonzalez and Woods, ${ }^{6}$ pp. 182-184) and the adaptive methods ${ }^{3,4}$ is that the adaptive methods use a neighborhood whose shape is determined by the actual pixel values. Although conceptually more elegant, this has the disadvantage of being computationally very expensive due to the morphological operations needed to determine the neighborhood shape.

\section{RESULTS OF METHODS DESCRIBED IN LITERATURE}

This section gives the results of the methods mentioned above. Based on these results a new method and its results are presented in the following sections.

The image used in this section is recorded with the TNO-FEL DUDA 1, a 12 bits 8-10 micron 1000x240 pixel camera. Figures 1-3 display the image with different settings used for the global stretching, corresponding to different setting of brightness and contrast. Figure 1 has settings such that the background and the upper part of the tank 


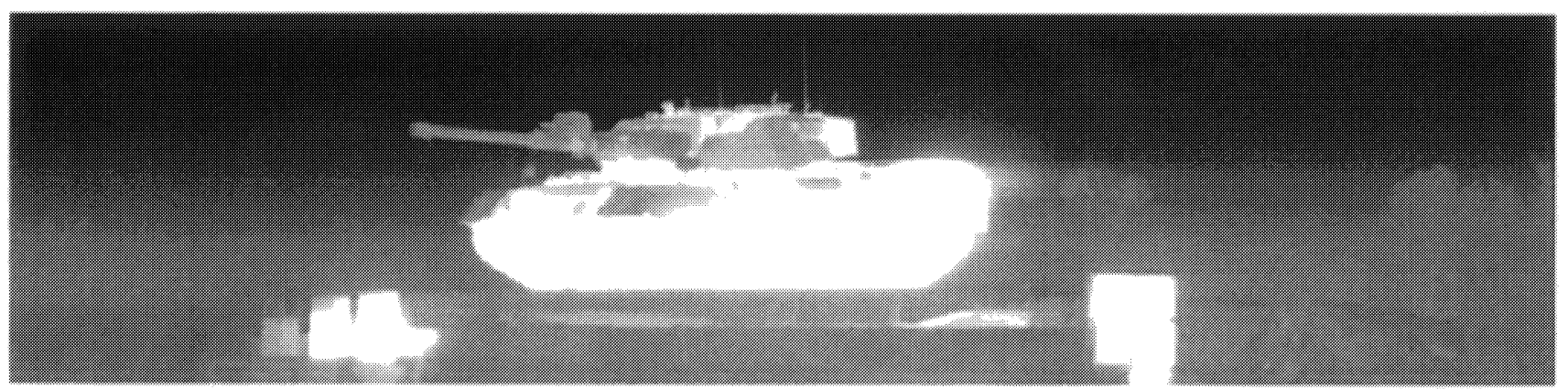

Figure 1. Image with first global stretch setting (ignore top and bottom $12 \%$ of histogram)

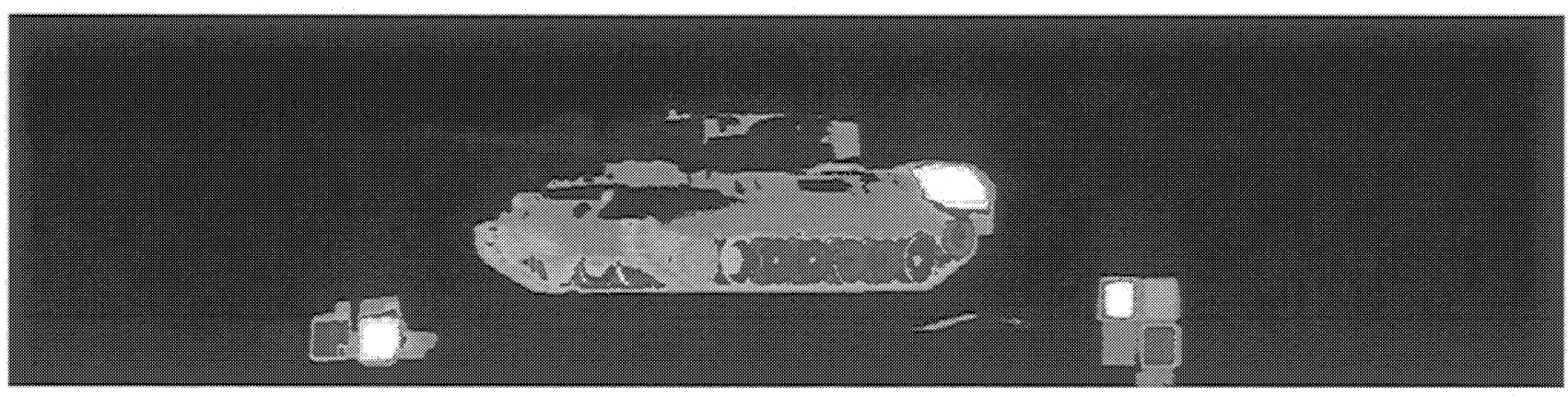

Figure 2. Image with second global stretch setting (ignore top and bottom $0.8 \%$ of histogram)

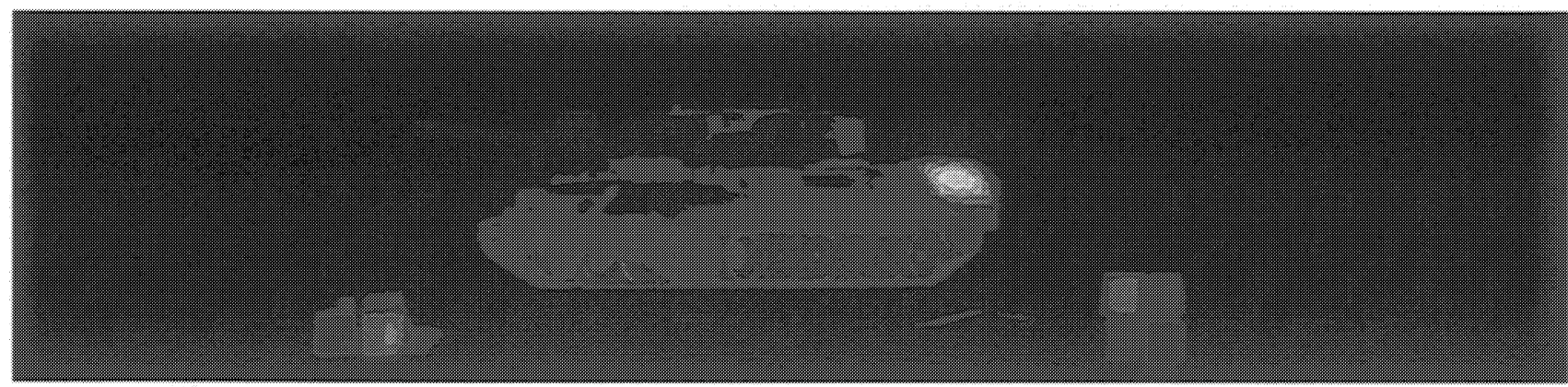

Figure 3. Image with third global stretch setting (include all pixels in histogram)

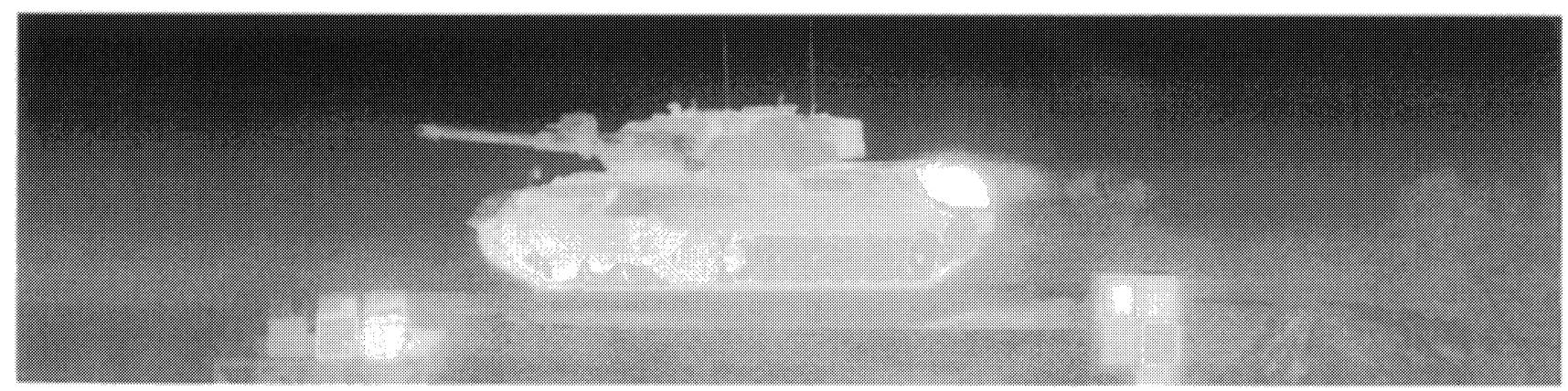

Figure 4. Histogram equalization 


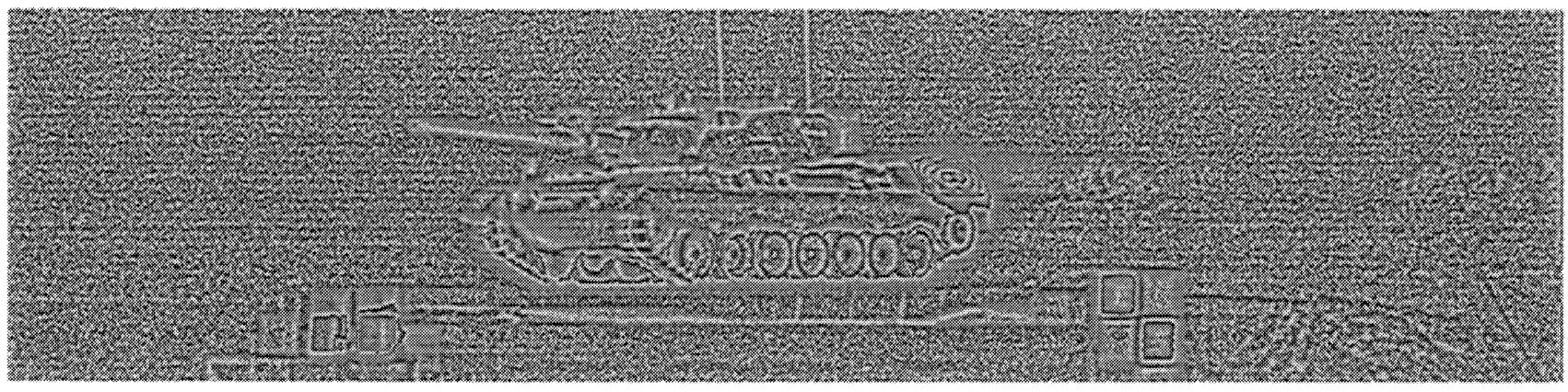

Figure 5. Local histogram equalization, using a $7 \times 7$ window

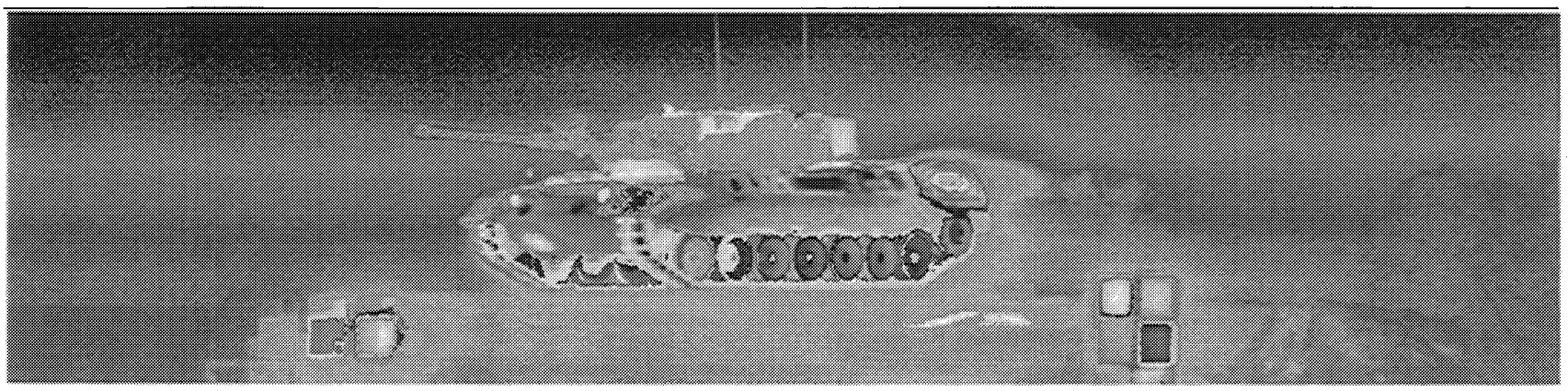

Figure 6. Adaptive local histogram equalization, using $t=16$ and $s=16$

are visible. In figure 2 the tracks and wheels are visible, and figure 3 gives the complete dynamic range of the image, thus showing the fact that the engine at the back is hot.

Figure 4 shows the result of histogram equalization applied to the image. As the foreground object is rather small compared to the cooler background, saturation occurs in the foreground.

Local histogram modification, shown in figure 5, produces an image in which small temperature differences are visible, but which also amplifies background noise. Also, any reference to absolute temperatures is lost.

Figure 6 shows the local adaptive histogram equalization. This method results in images where very much details are visible, but any temperature reference is lost; it is no longer obvious that the engine at the back is much warmer than the rest of the tank. Besides that this algorithm is exceptionally computational expensive.

Figure 7 shows the method as described by De Vries in Ref. 1. It gives quite some detail in the tracks, and suppresses the noise in the background. Comparing that with figure 8, depicting the method described by Narenda

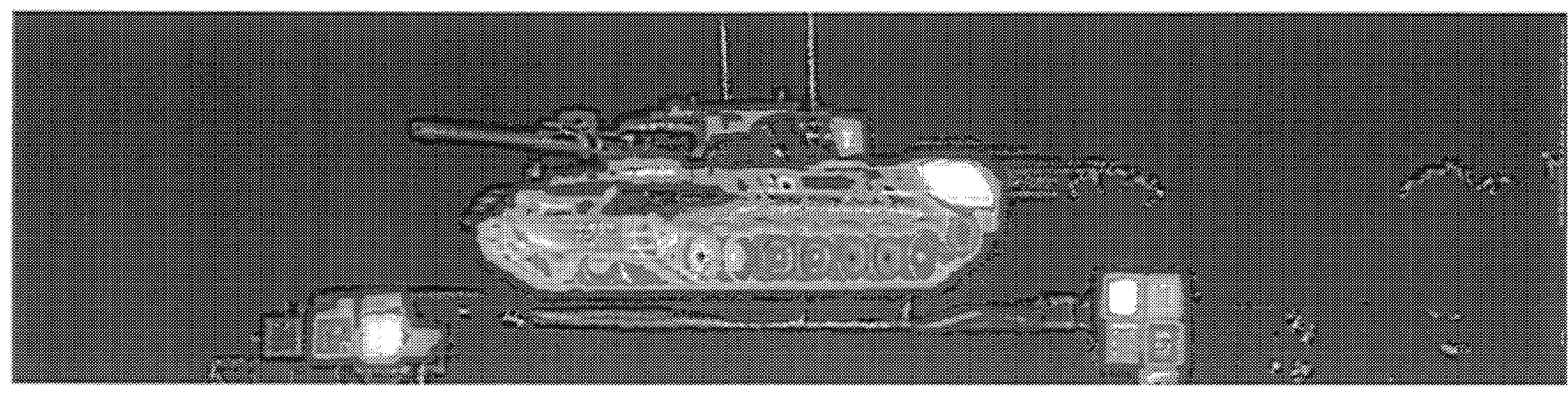

Figure 7. The De Vries method, using a $9 \times 9$ window, $\sigma_{n}=3$, and ignoring the top and bottom $1 \%$ of the histogram 


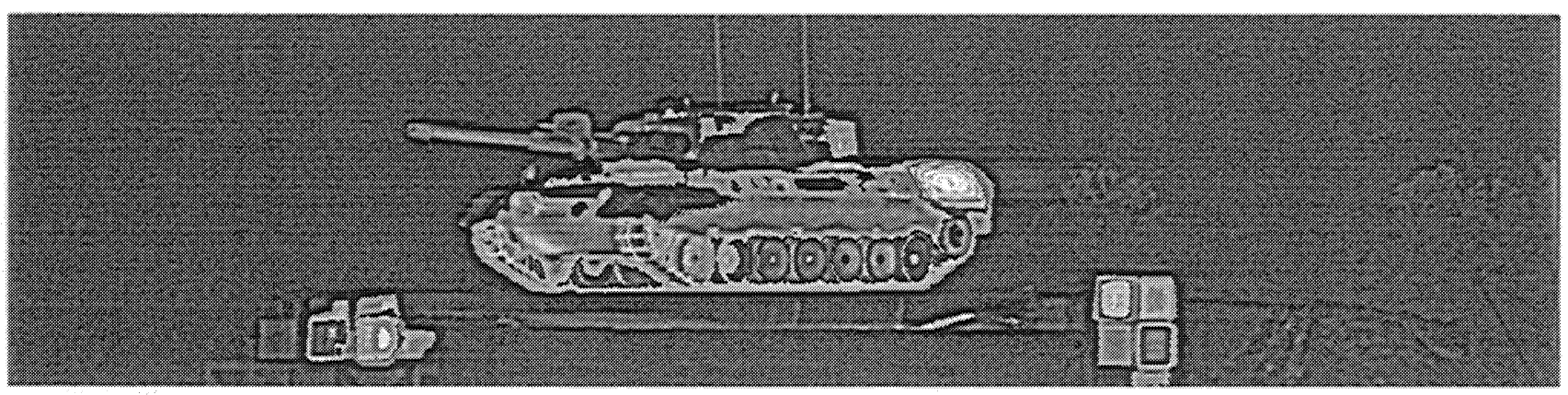

Figure 8. The method of Narenda and Fitch, using a $5 \times 5$ window, $\alpha=0.5, A_{\max }=200$, and ignoring the top and bottom $1 \%$ of the histogram

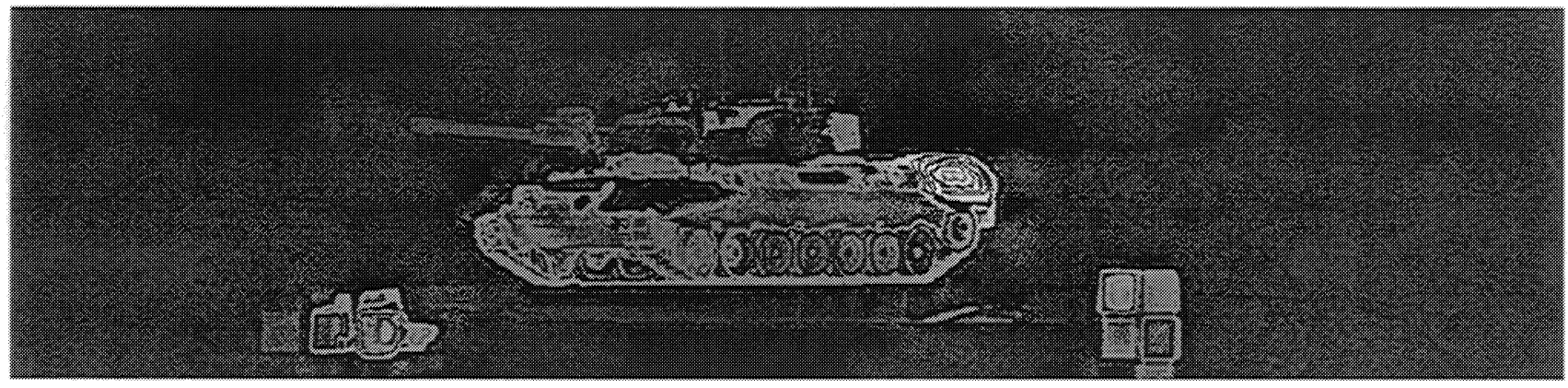

Figure 9. Adaptive multi-scale contrast enhancement by Toet

and Fitch in Ref. 2, we see quite some differences. Figure 8 does not show the artifacts occurring with the De Vries method caused by the noise threshold, at the expense of more background noise amplification.

The result of using the multi-scale method of Toet is depicted in figure 9. A wide temperature range is shown, including the antennas and the barrel of the tank, without extreme amplification of background noise. A disadvantage is that most details are transformed to a line drawing.

\section{AN IMPROVED METHOD}

Comparing the results presented in the previous section we see that different methods have different benefits. Using global stretch we only preserve the sense of cold and hot areas in the image, but there is no setting at which details in both background and foreground can be seen. Standard histogram equalization does not take into account that the highly interesting foreground occupies only a small part of the image, and thus of the histogram, resulting in a saturated view of the foreground. Local histogram equalization performs quite bad concerning background noise amplification. Adaptive local histogram equalization performs better regarding background noise, but lacks absolute temperature reference. The De Vries method raises problems regarding artifacts on low contrast areas. The Toet method tends to produce line drawings. Given the above, the most promising seems the method of Narenda and Fitch. It does not raise obvious artifacts, gives detail in both foreground and background, while maintaining absolute temperature reference.

A free parameter for many of the methods described is the scale at which windowing parameters are applied. The figures 10-13 show the results of the Narenda and Fitch method for window sizes 3x3, 9x9, 27x27 and $81 \times 81$. As we can see in these figures, it is hard to set an optimal scale. At small scale, we have enhancement of small details as can be seen in the tracks. At medium scale, we can see medium sized details as within the wheels. At large scale we can see large details such as the barrel. At very large scale the course vegetation outlines in the background are emphasized. 


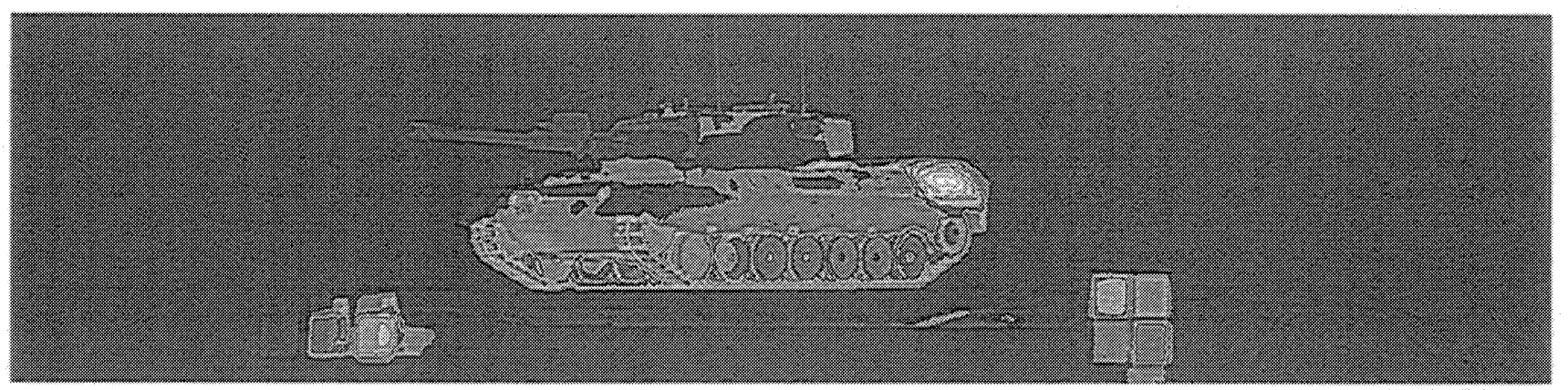

Figure 10. The method of Narenda and Fitch, using a $3 \times 3$ window, $\alpha=0.5, A_{\max }=100$, and ignoring the top and bottom $1 \%$ of the histogram

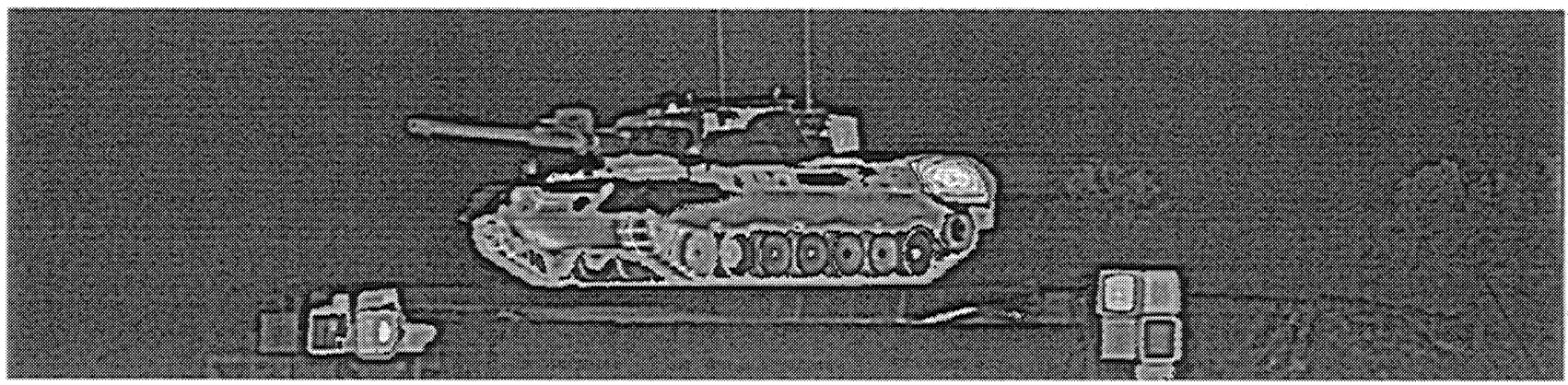

Figure 11. The method of Narenda and Fitch, using a 9x9 window, $\alpha=0.5, A_{\max }=100$, and ignoring the top and bottom $1 \%$ of the histogram

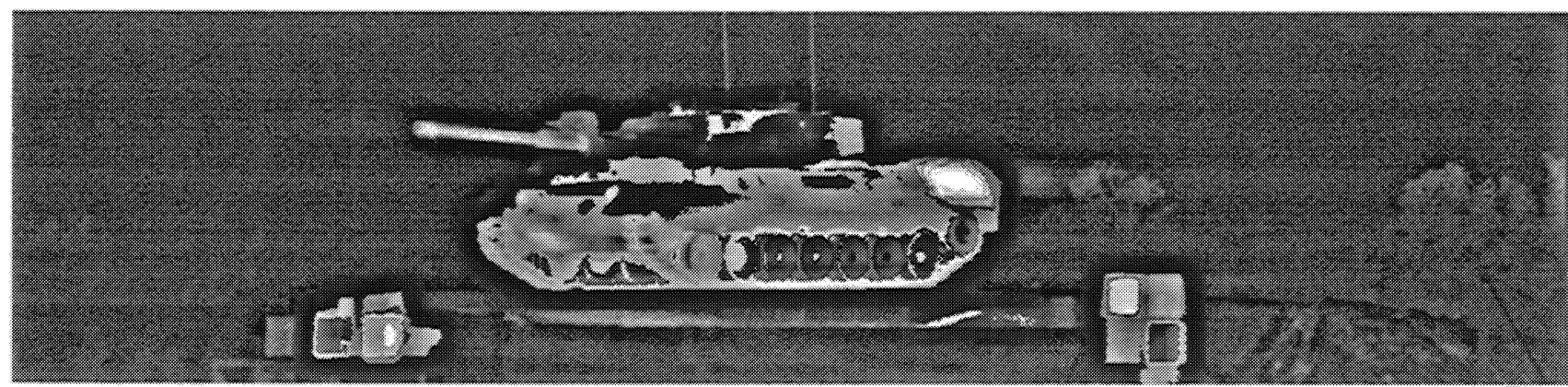

Figure 12. The method of Narenda and Fitch, using a $27 \mathrm{x} 27$ window, $\alpha=0.5, A_{\max }=100$, and ignoring the top and bottom $1 \%$ of the histogram 


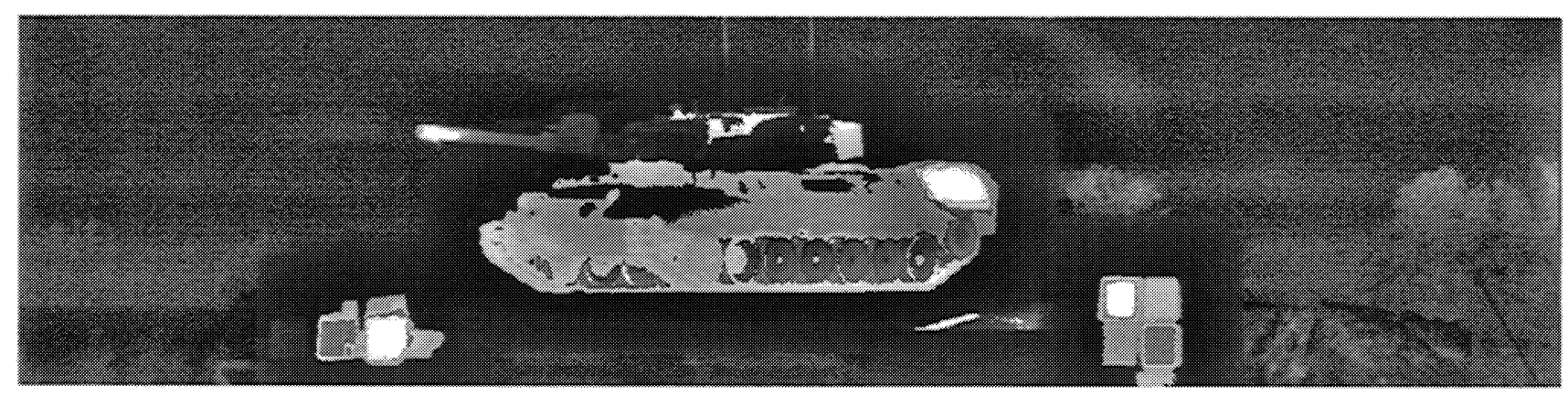

Figure 13. The method of Narenda and Fitch, using a $81 \times 81$ window, $\alpha=0.5, A_{\max }=100$, and ignoring the top and bottom $1 \%$ of the histogram

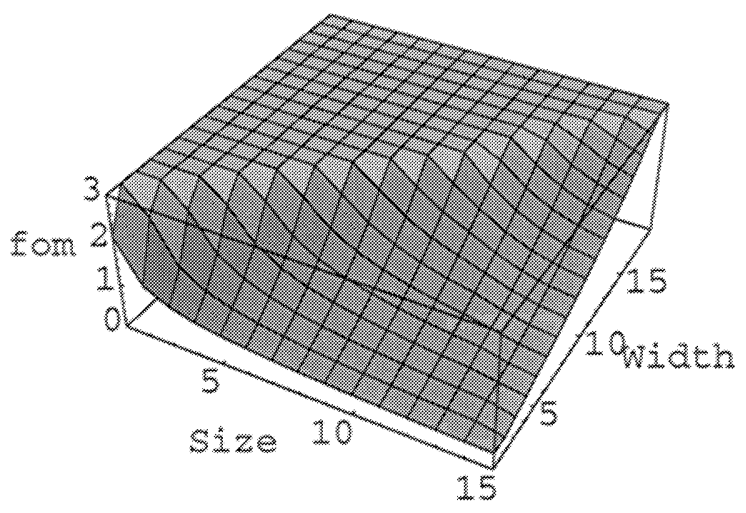

Figure 14. Fom as function of object size and window width. It is thresholded at fom $=3$.

\section{MULTI-SCALE APPROACH}

Last section showed that no single scale is optimal. This also is depicted in figure 14 and figure 15 . Figure 14 shows the amplification of an object of changing size for different scales. It depicts the figure of merit:

$$
\text { fom }=\frac{\bar{f}}{\sqrt{\sigma_{f}^{2}+\sigma_{b}^{2}}}
$$

with $\bar{f}$ the mean value of the (square) object, $\sigma_{f}$ the standard deviation in the object and $\sigma_{b}$ the standard deviation in the background changed by the filter process. A large fom means that the amplification of the object is large compared to the distortion produced by the amplification; a small fom means that the distortion is comparatively large compared to the amplification. Figure 15 depicts the relative amplification of a smaller and a larger object with respect to the scale. The smaller and the larger object are setup as depicted in figure 16. Using the above we can set up a multi scale scheme. In the following, $s$ is the size of the small object, $b$ the size of the larger object, and $w$ the window size of the scale under consideration. For a certain application a fom should be picked. If we take 1 as the minimal fom, then can be deduced from figure 14 that $w$ should at least exceed $s / 2$. If we have a multi-scale scheme where the $w$ 's used are the series $\left(3,3 m, 3 m^{2}, 3 m^{3}, \ldots\right)$, there is for any $s$ a smallest window $w$ still amplifying size $s$ :

$$
w \in[s / 2, m / 2 s]
$$




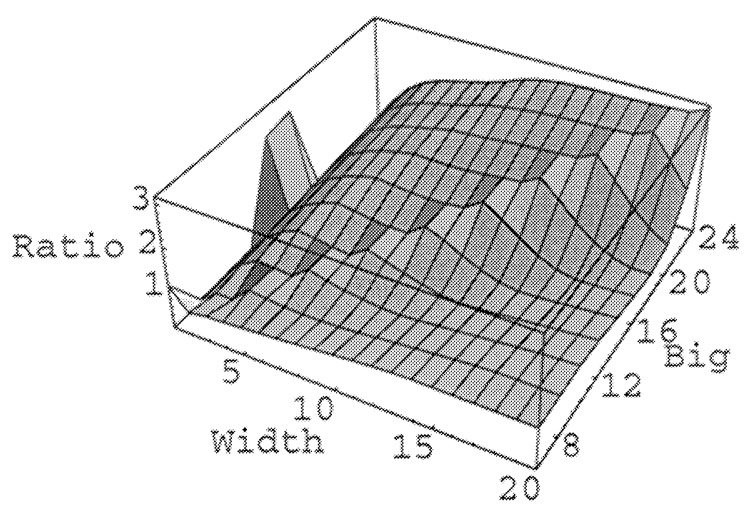

Figure 15. Ratio of amplification of smaller and larger object. The smaller object is $6 \times 6$ pixel, and the larger object's size is the axis Big. Width is the size of the window used in processing.

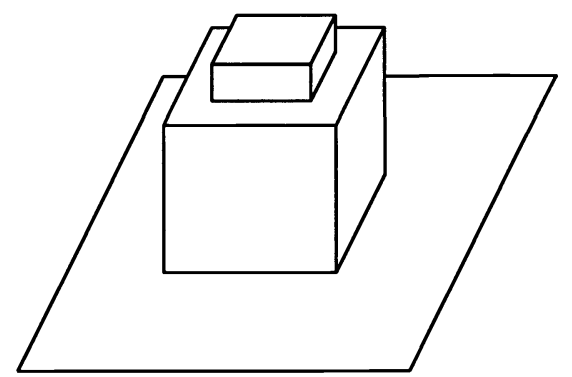

Figure 16. The intensity for the larger and the smaller object as used for figure 15. 


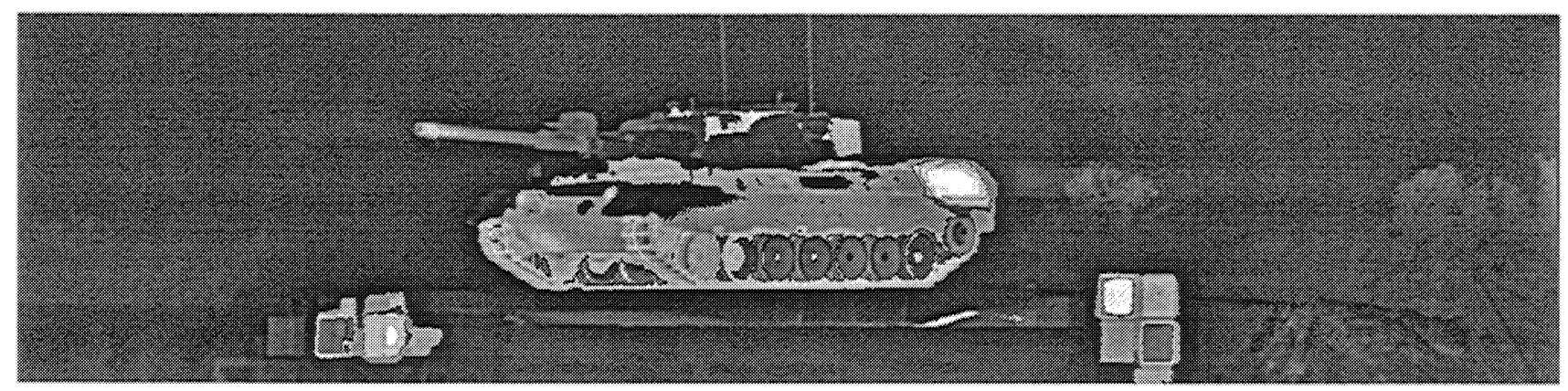

Figure 17. The proposed multi-scale method, using $3 \times 3,9 \times 9,27 \times 27$ and $81 \times 81$ windows, and ignoring the top and bottom $1 \%$ of the histogram

From figure 15 can be deduced that $b-s$ should be bigger then $w$ to get any significant output for the smaller object. This leads to

$$
b>[3 / 2 s, 1+m / 2 s]
$$

depending on the location of $s$ relative to the series of $w$. Now we should pick at what size ratio we want to be able to distinguish between larger and small objects. When 2.5 is taken, we get $b=2.5 s$. Using the upper side of the range in formula 4 , we get $2.5 s=1+m / 2 s$, or $m=3$.

\subsection{Combining scales}

Now we have set the number of levels in the multi-scale approach, we should consider the way to combine the processing at different scales. The specific version of formula 1 used by Narenda and Fitch is:

$$
o=\alpha \frac{M}{s}(i-m)+m=i+c(s) \cdot(i-m)
$$

with $M$ the global mean of the image, $s$ the local standard deviation $c(s)=\alpha \frac{M}{s}-1 . c(s)$ is clipped to $A_{m a x}$ on the top and to zero on the bottom of the range. As such the scheme of Narenda and Fitch applied at a certain scale is just an addition to the original image; a logical multi-scale extension is then also an addition to the original image:

$$
o=i+\sum_{i=1}^{k} c\left(s_{i}\right) \cdot\left(i-m_{i}\right)
$$

with $s_{i}$ and $m_{i}$ the local standard deviation and local mean at scale $i$. The result obtained at a combination of 4 scales $3,9,27,81$, is shown in figure 17 . It can be seen that the multi-scale approach results in an image showing simultaneously a large range of temperatures (from cold background to warm engine) and a large range of scales (from thin antennas to vegetation in the background), without producing artifacts such as found with different methods.

\subsection{Parameter settings}

The proposed multi-scale algorithm depends on the following parameters:

1. the number of scales used

2. the weighing factor $\alpha$ with the original image

3. the maximum multiplication allowed

The first parameter, the number of scales used, can be determined by the recipe involving the fom and the size ratio of smaller and larger objects given above. The second parameter $\alpha$ is application dependend: when the absolute temperature is more important $\alpha$ should decrease. The third parameter is related to the estimated noise of the imaging system, and thus a constant for a certain camera. 


\section{CONCLUSIONS}

The presented results show that multi-scale adaptive gain control can be used to obtain images showing all interesting features from high dynamic infrared imagery.

A new multi-scale method is presented for adaptive gain control which does not only show interesting features in all temperature ranges, but which also applies this process to different spatial scales.

A hardware implementation of the proposed multi-scale algorithm seems feasible.

\section{REFERENCES}

1. F. de Vries, "Automatic, adaptive, brightness independent contrast enhancement," Signal Processing 21, pp. 169$182,1990$.

2. P. M. Narenda and R. C. Fitch, "Real-time adaptive contrast enhancement," IEEE Transactions on Pattern Analysis and Machine Intelligence 3(6), pp. 655-661, 1981.

3. R. B. Paranjape, W. M. Morrow, and R. M. Rangayyan, "Adapative-neighborhood histogram equalization for image enhancement," Computer Vision, Graphics and Image Processing: Grahpical models and image processing 54(3), pp. 259-267, 1992.

4. D. Mukherjee and B. Chatterji, "Adaptive neighborhood extended contrast enhancement and its modifications," Graphical Models and Image Processing 57(3), pp. 254-265, 1995.

5. A. Toet, "Adaptive multi-scale contrast enhancement through non-linear pyramid recombination," Pattern Recognition Letters 11, pp. 735-742, 1990.

6. R. C. Gonzalez and R. E. Woods, Digital Image Processing, Addison-Wesley, 1992 (reprinted with corrections 1993). 